\title{
Medulloepithelioma, An Embryonal Tumor of the Central Nervous System, the Eye and the Optic Nerve: Review of the Clinical Behavior, Cytogenetic and Molecular Characteristics
}

\author{
Jamie Shoag ${ }^{1,2}$, Alyssa A Cavanaugh ${ }^{1}$, Jennifer Reichbach ${ }^{1}$, Joicy B Estevez ${ }^{2}$, Edward D Ziga ${ }^{1}$, \\ Antonello Podda ${ }^{1 *}$ \\ ${ }^{1}$ University of Miami, Leonard Miller School of Medicine, USA \\ ${ }^{2}$ Holtz Children's Hospital, Jackson Health System
}

*Corresponding author: Antonello Podda, University of Miami, Leonard Miller School of Medicine, USA,

Email: apodda@med.miami.edu

\section{ARTICLE INFO}

Received: 慧 March 04, 2020

Published: March 12, 2020

Citation: Jamie S, Antonello P. Medulloepithelioma, An Embryonal Tumor of the Central Nervous System, Eye and Optic Nerve: Review of the Clinical Behavior, Cytogenetic and Molecular Characteristics. Biomed J Sci \& Tech Res 26(3)-2020. BJSTR. MS.ID.004368.

\section{ABSTRACT}

Keywords: Tumors; Medulloepitheliomas; Neuroectodermal Tumors; Embryonal Tumors

Abbreviations: MEP: Medulloepitheliomas; CNS: Central Nervous System; WHO: World Health Organization; ETANTR: Embryonal Tumor with Abundant Neuropils and True Rosettes; ETMR: Embryonal Tumor with Multilayered Rosettes

\section{Introduction}

Medulloepitheliomas (MEPLs) are rare embryonal tumors characterized by histological features that resemble those of the neural tube, the embryonic precursor to the Central Nervous System (CNS). These tumors affect the CNS, the neuroepithelial structures of the eye, and the optic nerve, which are both a direct extension of the CNS. Their cell of origin is a primitive neuroectodermal cell, main cellular constituent of the neural tube. MEPLs originating in these anatomical sites have many similarities from the morphological standpoint, immunohistochemical profile and the cell of origin suggesting a close relation. On the other hand, they display many significant differences from the standpoints of their clinical behavior, and their molecular and epigenetic signatures suggesting that these tumors remain classified as distinct entities.

\section{Classification of CNS Embryonal Tumors}

In the 2016 World Health Organization (WHO) classification of
CNS tumors, the term Central Nervous System Primitive Neuroectodermal Tumors (CNS -PNET) is no longer in use [1]. CNS-PNET were described in the former 2007 WHO classification as a heterogenous group of CNS embryonal neoplasms composed of undifferentiated or poorly differentiated neuroepithelial cells [2]. With the discovery and integration of new molecular signatures, this entity has been replaced by multiple distinct embryonal tumors subtypes. Medulloblastomas are a more frequent, and distinct group of embryonal CNS tumors specifically originating in the cerebellum, that remain as entities separate from the other embryonal tumors former PNETs and will not be part of the topic in this review. Neuroectodermal cells are the most primitive progenitor cells of the nervous system. They constitute the wall of the neural tube in the early embryonic development [3]. These cells are multipotent with divergent differentiation capability towards the neuronal, astrocytic, and ependymal lines. Tumoral cells of the CNS -PNET group share these same characteristics. 


\section{CNS Embryonal Tumors (PNETs)}

In the previous classification, PNET with exclusive neuronal differentiation were termed Cerebral Neuroblastomas (NB). Those with predominant ependymal differentiation, characterized by the presence of ependymoblastic true rosettes (i.e. multilayered arrangements of mitotically active tumor cells around a central lumen) were termed Ependymoblastomas (EBL). Tumors predominantly composed by structures that recreate features of the primitive neural tube (i.e. tubular, papillary, trabecular, or even glandular-appearing arrangements of primitive cells often with an outer layer) were called Medulloepitheliomas (MEPL) [2,4]. Another rare, variant of CNS -PNET termed Embryonal Tumor with Abundant Neuropils and True Rosettes (ETANTR) is characterized by focal areas of high cellularity, broad bands of neoplastic neuropils and ependymoblastic rosettes [5]. ETANTR are very rare tumors associated with poor clinical outcomes $[2,5,6]$. Interestingly, all the histological features which traditionally characterize these CNS embryonal tumors, can be identified across all subtypes in different proportions [6,7].

Over the past decade, with the widespread use of molecular techniques, a new classification of embryonal CNS tumor has emerged. This system categorizes tumors by characteristic genetic alterations rather than histologic subtypes. In 2008, Pfister et al. first described a case of a child with an ETANTR with a novel focal amplification at 19q13.42 and up -regulation of the corresponding microRNAs (miRNAs) cluster [8]. Li et al. then detected the presence of an amplification of $19 q 13.41$ in $25 \%$ of 41 cases of CNS-PNETs analyzed [9]. The 19q13.41 amplified tumors were also found to be characterized by high-level of expression of the microRNAs cluster contained in the involved chromosomal segment, C19MC. The authors demonstrated that some of these miRNAs promote oncogenicity by modulating cell survival and enhancing growth of untransformed human neural stem cells in part by upregulating the WNT pathway signaling and restricting their differentiation [9]. In 2010, Korshunov et al. analyzed 41 tumor specimens morphologically diagnosed as EBLs or ETANTRs by FISH hybridization. Strikingly, this revealed the described 19q13.42 amplifications in 93\% of the samples [10]. The shared genetic signature of ETANTRs and EBLs suggested a common precursor origin of these tumors [6, 8,9]. Paulus and Kleihues suggested therefore introduction of the inclusive term Embryonal Tumor with Multilayered Rosettes (ETMR) to describe the new entity of 19q13.42 amplified tumors, given the ependymoblastic multilayered rosettes are the most significant common histological feature in both subtypes [6]. Subsequently, a subset of CNS MEPLs was also found to have this same molecular alteration and displayed ependymoblastic rosettes [2]. Thus, these neoplasms were also included under the ETMR terminology [11].

The most recent 2016 WHO classification groups the three neoplasms with the specific molecular signature (ETANR, EBLs and MEPLs) under the new entity "ETMR, C19MC-altered"[1]. Tumors with the typical histological features of an ETMR but that are either not tested for or do not display the C19MC molecular signature, are designated ETMR, NOS. MEPLs, neural-tube phenotype, that do not display C19MC retain the separate term Medulloepitheliomas. Amongst all CNS MEPLs, up to 25\% lack C19MC amplification [12]. Presently, these CNS MEPLs that are not C19MC-altered have unclear genetic underpinnings.

\section{Intraocular MEPL}

Intraocular Medulloepitheliomas (IO-MEPL) share the same cellular origin of CNS MEPLs, ETMRs and other embryonal CNS neoplasms, the primitive neuroectodermal cell [13]. The neural components of the eye originate during the embryonal development in the 4-week embryo after the neural tube closes, by an evagination in its cephalic end rostral portion (the forebrain) called the optic vesicle. The optic vesicles come in contact with the surface of the ectoderm inducing changes necessary for lens formation. Shortly thereafter, the optic vesicle begins to invaginate forming the double-walled optic cup. The outer layer of the optic cup forms the pigmented layer of the retina, the outer layers of the ciliary bodies, and the iris. The inner or neural layer of the optic cup differentiates into the light receptors of the retina, the inner layer of the iris (pars iridica retinae), and of the ciliary bodies (pars ciliaris retinae) [3]. Intraocular Medulloepitheliomas (IO-MEPL) originate via a transformation of this primitive medullary epithelium of the inner layer of the optic cup [13]. Each of the common locations of the IO-MEPLs represents the places where the closure the optic cup and optic stalk fetal fissure (choroid fissure) last occurs and where the final differentiation of the last multipotent neuroepithelial progenitor cells takes place [14].

The overwhelming majority of IO-MEPL originate from this nonpigmented epithelium in the ciliary bodies. These tumors are called ciliary body MEPLs, formerly known as diktyomas because of their net-like histological appearance [15]. Rarely, IO-MEPL originate from the primitive medullary epithelium in the iris, the retina or the optic nerve [2]. Optic nerve Medulloepitheliomas (ON-MEPLs) can originate at the levels of the optic disk or the extraocular portion of the nerve $[2,14]$. IO-MEPL are the second most common primary intraocular neoplasm of childhood after retinoblastoma [16]. On the other hand, the ON-MEPLs subtype are extremely rare with only approximately 10 cases reported in the international literature.

\section{CNS and Ocular MEPLs Differences}

Although histologically similar, ocular MEPLs, ETMR, and CNS MEPLs have very significant differences. Patients with ETMR are usually very young, the majority occurring in the first 2 years of life and these tumors have never been described in the adult population $[14,17]$. Conversely, ocular MEPLs have been reported throughout the lifespan, with approximately $15-20 \%$ of cases occurring in adults [18]. These discrepancies in age distribution already imply a 
fundamental difference in the histogenesis and underlying molecular mechanisms [14]. The prognosis of ocular MEPLs is significantly better than that of their cerebral counterparts. Among ocular-MEPLs, those originating from the ciliary bodies have the best prognosis. IO-MEPLs have an excellent survival after enucleation (nearly $100 \%$ if no extraocular extension) in comparison to the almost-uniformly fatal CNS counterpart. Optic nerve MEPLs have an intermediate behavior between those of the ciliary bodies and those of the CNS given the propensity to extend into the orbit towards the leptomeninges [1]. Intraocular MEPLs are very small at diagnosis being symptomatic and detected usually in early stages of their formation [14]. Their greatest diameter has been reported being no more than $2 \mathrm{~cm}$ even when diagnosed late and the entire eye is filled with tumor, due to the presence of a natural barrier to spread such as the sclerocornea. Other known biological factors common in these tumors like the LIN28A overexpression, a known marker for malignancy, are not believed to supersede these anatomical factors governing metastasis and influence the clinical outcome of IOMELP entirely confined to the globe [14]. MEPLs originating in the posterior pole from the optic disk and the extraocular portion of the optic nerve lack this natural anatomic barrier against spread with a potential risk to course the optic nerve and spread to the CNS.

Intraocular Medulloepitheliomas can be characterized as "teratoid or non-teratoid." This classification is based on the presence or absence of heteroplastic elements (i.e. hyaline cartilage, muscle, myoblasts, and brain-like tissue). No such morphological distinctions exist for the CNS counterpart [19]. Approximately 70\% of ocular Medulloepitheliomas are classified as malignant [18]. Among ophthalmic pathologists it is agreed that the separation of benign from malignant intraocular Medulloepitheliomas can be notoriously difficult [14]. Because the current WHO ocular tumors classification criteria for malignancy does not correlate with clinical behavior or histologic appearance, a new simple grading scheme has been proposed by Verdijk, to replace the current dichotomy $[18,20]$. The scheme comprises grade I tumors (corresponding to the current WHO designation "benign"), grade II tumors (with signs of progression like pleomorphism, increased mitotic activity, and local invasion), and grade III tumors (with malignant transformation and metastatic potential, evidenced by extra scleral extension or metastasis). Metastatic disease occurs only in cases with extraocular extension $[18,20]$.

\section{Molecular biology of ETMR and Medulloepitheliomas}

A large percentage of CNS ETANR, EBLs and MEPLs are characterized by the presence of an amplification of 19q13.4 and subsequent high-level of expression of the microRNAs cluster contained in the involved chromosomal segment, C19MC (the so called "ETMR, C19MC-altered" entity). Some of these miRNAs promote oncogenicity by modulating cell survival and enhancing growth of untransformed human neural stem cells in part by upregulating WNT pathway [1,9]. Tumors with the typical histological features of an
ETMR but that are either not tested for or do not display the C19MC molecular signature, are designated ETMR, NOS. The $25 \%$ of CNS MEPLs, the neural-tube phenotype, that do not display C19MC [12] retain the separate term Medulloepitheliomas of the CNS. Presently, these CNS MEPLs that are not C19MC-altered have unclear genetic underpinnings. The $\mathrm{C} 19 \mathrm{MC}$ molecular signature has never been described in ocular-MEPLs. This group of embryonal CNS and ocular tumors entities seem to share many similarities in their immunoprofiles [19]. This finding and the similar histological features suggested a relation between these ocular and CNS tumors which is consistent with a shared embryologic origin from the primitive medullary epithelium of the neural tube [19]. Interestingly, regardless of their morphologic pattern, ETMR NOS, C19MC-altered ETMR of the CNS often show a strong LIN28A protein immunoreactivity in some portions of the tumor [21]. This marker is therefore considered, although not entirely specific, a useful surrogate marker for the diagnosis of these tumors [21]. LIN28A may be also expressed by CNS Medulloepitheliomas that do not have C19MC amplification [21] and by almost all ocular Medulloepitheliomas. The LIN28A intensity of staining has been shown to correlate with aggressive behavior $[18,14]$.

LIN28A is considered a marker for malignancy in both CNS and ocular tumors. LIN-28 family RNA-binding protein acts as a posttranscriptional regulator of genes involved in developmental timing and self-renewal in embryonic stem cells, through direct interaction with target mRNAs and by disrupting the maturation of certain miRNAs involved in embryonic development. miRNAs are short, approximately 20-30 nucleotide sequences of non -coding RNA which function as important post -transcriptional regulators of gene expression in various cell types mostly by binding specific messenger RNA (mRNA) targets via direct base-pairing interactions [22]. miRNAs are transcribed initially as large RNA precursors the pri-miRNAs that are then processed in the nucleus by the RNase enzyme, Drosha, and RNA-binding protein, Pasha into 70-nucleotide pre-miRNAs, which fold into imperfect stem-loop structures. The pre-miRNAs are then exported into the cytoplasm by a specific transporter and undergo an additional processing step in which a double -stranded RNA of $\sim 22$ nucleotides in length, is excised from the pre-miRNA hairpin by another RNAse called Dicer [22]. The mature miRNA single strand is then preferentially retained in the functional complex that regulates its target genes' mRNA.

miRNAs are implicated in many biological processes including self-renewal and pluri-potency of embryonal stem cells [22]. A group of miRNAs named oncomirs, have been implicated as regulators of the expression of genes involved in several human malignancies [23]. Through alteration of their expression in various cancers, oncomirs miRNAs function indirectly as both tumor suppressors and oncogenes downregulating, or upregulating different pathways involved in oncogenesis [23]. Patterns of miRNA expression have been shown to distinguish tumor types and to predict tumor bi- 
ology [24]. Other components of the miRNA-machinery have also been implicated in tumorigenesis, for example the alteration of the expression of the regulatory protein Dicer has been shown to be downregulated, among others, in some cases of lung cancer with the finding correlating with shortened survival [23]. Although miRNAs have been implicated in malignant gliomas [25], their role in malignant pediatric CNS tumors remained largely unexplored until these more recent discoveries of their involvement in ETMRs [9].

Another example of oncomir is lethal-7 (Let-7) a member of a highly conserved homonymous miRNA family [26]. The let-7 family is known to function as a tumor suppressor by down-regulating the Ras oncogenes, a family of genes involved in cellular growth and differentiation found to be mutated in about $15-30 \%$ of human tumors. Several groups demonstrated a reduced expression of the microRNAs in human cancers. and its association with shortened survival. $[22,25]$. The strong expression and immunoreactivity of LIN28A protein in some portions of the tumor is characteristic of all these embryonal CNS and ocular tumors [27]. LIN-28 family of RNA-binding protein acts as a posttranscriptional regulator of genes through direct interaction with target mRNAs and by disrupting the maturation of certain miRNAs. LIN28A prevents the terminal processing of the LET7 family of microRNAs.

Despite several similarities, IO-MEPL, CNS ETMR and MEPL present several other significant biological differences. Copy-number aberrations (CNAs) have been studied in several series in both ocular and CNS tumors. The median number of CNAs was significantly higher in IO-MEPL 6.8 per tumor vs. 2.3 per tumor ETMR (P $<0.01$ ) [19]. Interestingly the median number of CNAs was significantly lower in IO MEPL diagnosed in adults (2.2 per tumor vs. 7.2 per tumor for children; $\mathrm{P}<0.01$ ) [19]. The genetic hallmark aberration of ETMR, the amplification of 19q13.42 C19MC locus often in conjunction with complex intra-chromosomal rearrangements in or around the 19q13 locus, was not identified in any IO MEPL [19]. Also gain of chromosome 2, frequently present in ETMR (80\%), was found only in 30\% of IO-MEPL. Cytogenetic analysis of ocular tumors often shows numerous others recurrent CNAs involving either whole chromosomes or chromosome arms, that are absent in ETMRs. Loss of $1 p, 4,16 p$ or gain of 8 that were the most frequently detected CNAs in IO-MEPL. Isochromosome 1q (simultaneous loss of $1 p$ and gain of $1 q$ ) was detected in approximately $15 \%$ of 10 MEPL cases.

Intraocular Medulloepitheliomas has been rarely described in patients with DICER1 syndrome or pleuropulmonary blastoma (PPB) familial tumor and dysplasia syndrome caused by germline mutations in DICER1. The syndrome is characterized by pleuropulmonary blastoma of the lungs and pleura (which in its solid form can metastasize), cystic nephroma, Sertoli-Leydig cell neoplasms, multinodular goiter, and rarely IO-MEPL. DICER1 syndrome is an autosomal dominant trait with variable penetrance and most affected individuals do not develop the full spectrum of the disease.
Approximately $3 \%$ of all IO-MEPLs are associated with this syndrome and less than 1\% of PPB patients develop IO-MEPL [16]. Most mutations in DICER1 are truncating with loss of function and consequent dysregulation miRNA biogenesis.

Sahm et al. [16] analyzed the mutational landscape, epigenetic profiles and copy-number aberrations (CNAs) of 19 IO-MEPL specimens. They identified recurrent mutated genes (i.e. present in 2 or more samples) in 18 of the 19 tumors analyzed. The most common mutations detected involved DICER1 in 32\%, and KMT2D (formerly known as MLL2) in 26\% of cases. These two frequent somatic mutations in IO-MEPL were mutually exclusive and taken together involved almost $60 \%$ of the tumors. Other recurrent mutations identified at a lower frequency were BRCA2 (21\%), NOTCH2 (16\%), BRCA1 (11\%), CDH1 (11\%), GSE1 (11\%), PTCH1 (11\%), FGFR1 (11\%), and KDR (11\%) and were distributed with various combinations.[16] ARID1B gene was mutated in 2/19 tumors (11\%) and ARID2 in 1 tumor. Within DICER1, almost all mutations were within exons 24 and 25 which code for the functional RNAse catalytic domains and were found across all ages [16]. The authors also analyzed the DICER1 gene in a series of 20 intracranial ETMR with or without the C19MR alteration and found only one post fossa ETMR with no C19MR displaying a mutation of DICER1 [16]. No associations between specific mutations and tumor behavior was detected [16].

Mutually exclusive somatic mutations of DICER1 and KMT2D are frequent in intraocular Medulloepitheliomas of the ciliary body, but posterior lesions of the eye (involving the optic disk or the optic nerve) have not been fully analyzed [25]. MEPL of the optic nerve has not been reported associated with germline DICER Syndrome [25]. MicroRNA profiling in intraocular Medulloepitheliomas shows markedly upregulated or downregulated microRNAs. The microRNA dysregulation has profiles similar to those seen in glioblastomas and retinoblastomas [26]. The second most commonly mutated gene in IO-MPL is KMT2D a gene that encodes a histone methyltransferase 16. The mutations in KMT2D were interspersed throughout the gene and were detected only in children [16]. Unsupervised hierarchical clustering of DNA methylation has shown that intraocular Medulloepitheliomas have also signatures distinct from those of intracranial Medulloepitheliomas and are therefore clearly epigenetically distinct tumor entities [26,27]. The targetable epigenetic tumor protein EZH2 is enriched in intraocular Medulloepitheliomas [18].

\section{Conclusion}

Medulloepitheliomas of the CNS, the eye and the optic nerve are rare embryonal tumors characterized by histological features that resemble the neural tube. These tumors are similar to other embryonal tumors of the CNS grouped under the term or ETMR (C19MC altered or not) from the histological, immunohistochemical and embryological stand point since all seem to derive from a 
common primordial precursor cell of the CNS, the undifferentiated neuroepithelial cell of the neural tube, suggesting a close relation of these entities. However, theses embryonal tumors also display many significant differences in clinical behavior, and molecular and epigenetic signatures suggesting that they should be classified as distinct neoplasms. This group of tumors interestingly also seem to converge and share common terminal oncogenic mechanisms that ultimately seem to reinforce their close relation: the disruption of oncogenic miRNAs, oncomirs. miRNA are implicated in many biological processes including self-renewal and pluri-potency of embryonal stem cells [21]. These small RNAs are major regulators of cellular growth and differentiation and their aberrant expression is

often associated with several types of cancer and with cancer progression in multiple tissues. Further molecular investigations are necessary to better characterize these entities.

\section{Acknowledgements}

None.

\section{Conflict of Interest}

None.

\section{References}

1. Louis DN, Perry A, Reifenberger G, von Deimling A, Figarella Branger D, et al. (2016) The 2016 World Health Organization Classification of Tumors of the Central Nervous System: a summary. Acta Neuropathol, 131(6): 803-820.

2. Louis DN, Hiroko Ohgaki, Otmar D Wiestler, Webster K, Cavenee, et al (2017) The 2007 WHO classification of tumours of the central nervous system. Acta neuropathologica 114(2): 97-109.

3. Salder T, Langman's Medical Embryology. $11^{\text {th }}$ edn.

4. Phillips J, T Tihan, G Fuller (2015) Practical Molecular Pathology and Histopathology of Embryonal Tumors. Surgical Pathology Clinics 8(1): 73-88.

5. Eberhart CG, Brat DJ, Cohen KJ, Burger PC (2000) Pediatric neuroblastic brain tumors containing abundant neuropil and true rosettes. Pediatr Dev Pathol 3(4): 346-352.

6. Korshunov A, Sturm D, Ryzhova M, Hovestadt V, Gessi M, et al. (2014) Embryonal tumor with abundant neuropil and true rosettes (ETANTR), ependymoblastoma, and medulloepithelioma share molecular similarity and comprise a single clinicopathological entity. Acta Neuropathol 128(2): 279-289.

7. Paulus W, Kleihues (2010) Genetic profiling of CNS tumors extends histological classification. Acta Neuropathol 120(2): 269-270.

8. Stagner AM, FA Jakobiec (2016) Updates on the Molecular Pathology of Selected Ocular and Ocular Adnexal Tumors: Potential Targets for Future Therapy. Seminars in Ophthalmology 31(1-2): 188-196.

9. Pfister S, Remke M, Castoldi M, Bai AH, Muckenthaler MU, et al. (2009) Novel genomic amplification targeting the microRNA cluster at 19q13.42 in a pediatric embryonal tumor with abundant neuropil and true rosettes. Acta Neuropathol 117(4): 457-464.
10. Li M, Lee KF, Lu Y, Clarke I, Shih D, et al. (2009) Frequent amplification of a chr19q13.41 microRNA polycistron in aggressive primitive neuroectodermal brain tumors. Cancer Cell 16(6): 533-546.

11. Korshunov A, Remke M, Gessi M, Ryzhova M, Hielscher T, et al. (2010) Focal genomic amplification at 19q13.42 comprises a powerful diagnostic marker for embryonal tumors with ependymoblastic rosettes. Acta Neuropathol 120(2): 253-260.

12. Chiang JC, DW Ellison (2017) Molecular pathology of paediatric central nervous system tumours. J Pathol 241(2): 159-172.

13. Shields JA, SC (2008) Intraocular tumors. An Atlas and Textbook. $2^{\text {nd }}$ edn.

14. Jakobiec FA, Kool M, Stagner AM, Pfister SM, Eagle RC, et al. (2015) Intraocular Medulloepitheliomas and Embryonal Tumors with Multilayered Rosettes of the Brain: Comparative Roles of LIN28A and C19MC. Am J Ophthalmol 159(6): 1065-1074.

15. Canning CR, AC McCartney, J Hungerford (1988) Medulloepithelioma (diktyoma). Br J Ophthalmol 72(10): 764-767.

16. Sahm F, Jakobiec FA, Meyer J, Schrimpf D, Eberhart CG, et al. (2016) Somatic mutations of DICER1 and KMT2D are frequent in intraocular medulloepitheliomas. Genes, Chromosomes and Cancer 55(5): 418-427.

17. Perry A, J Brat (2017)Practical Surgical Neuropathology: A Diagnostic Approach $2^{\text {nd }}$ edition.

18. Grossniklaus HE, C Eberhart, T Kivela (2018) WHO Classification of Tumours of the Eye. $4^{\text {th }}$ Edition.

19. Korshunov A, Jakobiec FA, Eberhart CG, Hovestadt V, Capper D, et al. (2015) Comparative integrated molecular analysis of intraocular medulloepitheliomas and central nervous system embryonal tumors with multilayered rosettes confirms that they are distinct nosologic entities. Neuropathology 35(6): 538-544.

20. Verdijk RM (2016) On the Classification and Grading of Medulloepithelioma of the Eye. Ocul Oncol Pathol 2(3): 190-193.

21. Arie Perry, Daniel J Brat (2010) Practical Surgical Neuropathology A Diagnostic Approach. $1^{\text {st }}$ Edition.

22. Bar M, Wyman SK, Fritz BR, Qi J, Garg KS, et al. (2008) MicroRNA discovery and profiling in human embryonic stem cells by deep sequencing of small RNA libraries. Stem Cells 26(10): 2496-2505.

23. Esquela Kerscher A, Slack FJ (2006) Oncomirs - microRNAs with a role in cancer. Nat Rev Cancer 6(4): 259-269.

24. Elcie Chan, Daniel Estévez Prado, Joanne Barnes Weidhaas (2011) Cancer microRNAs: from subtype profiling to predictors of response to therapy. Trends Mol Med 17(5): 235-243.

25. Steven M Johnson, Helge Grosshans, Jaclyn Shingara, Mike Byrom, Rich Jarvis, et al. (2005) RAS Is Regulated by the let-7 MicroRNA Family. Cell 120(5): 635-647.

26. Farzaneh M, Attari F, Khoshnam SE (2017) Concise Review: LIN28/ let-7 Signaling, a Critical Double-Negative Feedback Loop During Pluripotency, Reprogramming, and Tumorigenicity. Cell Reprogram 19(5): 289-293.

27. Thales Papagiannakopoulos, Alice Shapiro, Kenneth S Kosik (2008) MicroRNA-21 Targets a Network of Key Tumor-Suppressive Pathways in Glioblastoma Cells. Cancer Res 68(19): 8164-8172. 
ISSN: 2574-1241

DOI: $10.26717 /$ BJSTR.2020.26.004368

Antonello Podda. Biomed J Sci \& Tech Res

(C) This work is licensed under Creative

Submission Link: https://biomedres.us/submit-manuscript.php

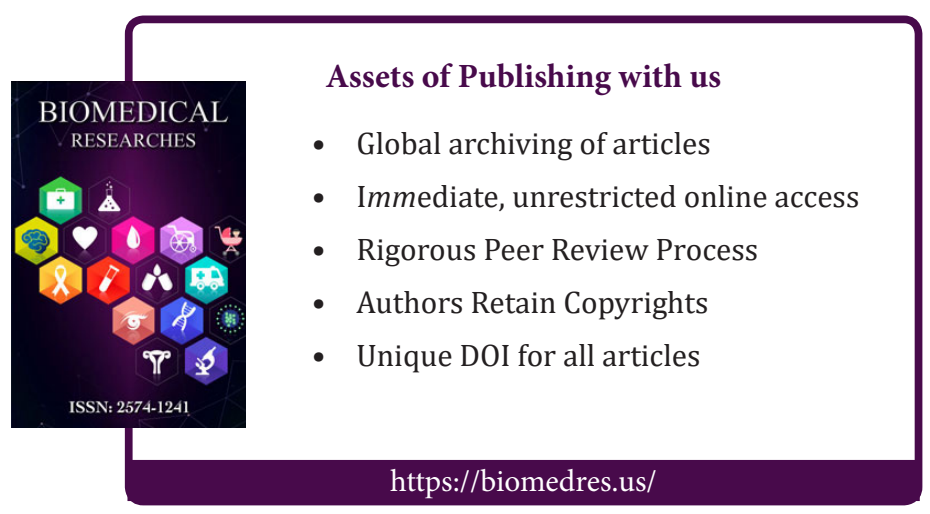

